\title{
The ChaOtic Motion OF THE Solar SYSTEM
}

\author{
J. Laskar \\ Bureau Des Longitudes, 77 Ave. Denfert Rochereau, 75014 Paris, France
}

\section{ABSTRACT}

In a previous paper (Laskar, Nature, 338, 237-238), the chaotic nature of the solar system excluding Pluto was established by the numerical computation of the maximum Lyapunov exponent of its secular system over $200 \mathrm{Myr}$. In the present an explanation is given for the exponential divergence of the orbits: it is due to the transition from libration to circul ation of the critical argument of the secular resonance $2\left(g_{4}-g_{3}\right)-\left(s_{4}-s_{3}\right)$ related to the motions of perihelions and nodes of the Earth and Mars. An other important secular resonance is identified: $\left(g_{1}-g_{5}\right)-\left(s_{1}-s_{2}\right)$. Its critical argument stays in libration over $200 \mathrm{Myr}$ with a period of about $10 \mathrm{Myr}$ and amplitude from $85^{\circ}$ to $135^{\circ}$. The main features of the solutions of the inner planets are now identified when taking these resonances into account. Estimates of the size of the chaotic regions are determined by a new numerical method using the evolution with time of the fundamental frequencies. The size of the chaotic regions in the inner solar system are large and correspond to variations of about $0.2 \mathrm{arcsec} /$ year in the fundamental frequencies. The chaotic nature of the inner solar system can thus be considered as robust against small variations of the initial conditions or of the model. The chaotic regions related to the outer planets frequencies are very thin except for $g_{6}$ which present variations sufficiently large to be significant over the age of the solar: system. 